\title{
How do interactive maternal traits and environmental factors determine offspring size in Daphnia magna?
}

\author{
F. Gabsi ${ }^{1 *}$, D. S. Glazier ${ }^{2}$, M. Hammers-Wirtz ${ }^{3}$, H. T. Ratte ${ }^{1}$ and T. G. Preuss ${ }^{1}$ \\ 1 Institute for Environmental Research, RWTH Aachen University, Worringerweg 1, 52072 Aachen, Germany \\ 2 Department of Biology, Brumbaugh Academic Center, Juniata College Huntingdon, Pennsylvania 16652, USA \\ 3 Research Institute for Ecosystem Analysis and Assessment (gaiac), Kackertstrasse 10, 52072 Aachen, Germany
}

Received 16 July 2013; Accepted 5 November 2013

\begin{abstract}
In this study, we investigated variation in offspring size (OS) of Daphnia magna in relation to multiple maternal traits and environmental variables. Data originated from laboratory experiments conducted at different feeding scenarios. The mother daphnids had different life-history traits and were reared under various feeding and density conditions. OS showed linear relationships with maternal traits, varying positively with maternal body size, age and brood number, and negatively with brood size and with the amount of ingested carbon. OS increased exponentially with crowding. Using stepwise multiple regression analysis, we developed an empirical model for the OS variation with the relevant maternal and environmental variables. Density dependence was considered by multiplying the resulting model by a density-effect function. We found that the ingested carbon and the maternal body size were the strongest determinants of the observed variation in the OS, whereas the brood size had the least impact on OS. Additionally, the brood number had no significant effect in determining the variability in the OS. The validity of the multivariate model was tested against an independent dataset. The model accurately predicted the OS despite several genetic and environmental differences compared with the data used for parameterization.
\end{abstract}

Key words: Daphnia magna / offspring size / maternal traits / maternal environment / modelling

\section{Introduction}

Owing to its essential role in food-web dynamics of freshwater ecosystems and to its importance as a test organism in ecotoxicology, the cladoceran Daphnia magna (Straus) is frequently used in modelling studies to answer diverse questions on the biology and ecology of this organism (Kooijman, 2000; Vanoverbeke, 2008; Preuss et al., 2009), or to elucidate complex mechanisms behind its responses to natural (Gergs et al., 2013) or anthropogenic (Preuss et al., 2010) stressors. Depending on the addressed question, ecological models can, in general, be based on empirical description of processes (Preuss et al., 2009) or on energetic concepts (Kooijman, 2000; Vanoverbeke, 2008). However, an important limitation of these available models is that none of them accounts for the natural variability in the offspring size (OS) in Daphnia magna. This trait, in fact, determines vital individual processes such as growth (Hammers-Wirtz and Ratte, 2000), size at first reproduction (Barata and Baird, 1998) or size-selective

\footnotetext{
*Corresponding author: faten.gabsi@bio5.rwth-aachen.de
}

predation (Lampert, 1993; Gergs and Ratte, 2009), which in turn affect higher-order processes such as population growth rate, maturation (Sakwinska, 2004; Rinke, 2006), population survival rates (Sakwinska, 2004; Dudycha and Lynch, 2005) or resistance to starvation (Tessier and Consolatti, 1991).

To efficiently capture the variation in the OS in Daphnia, it is essential to simultaneously account for the relevant variables that induce a change in this life history trait. Changes in OS in Daphnia are frequently a consequence of maternally mediated responses to environmental effects. As explained by Mousseau and Fox (1998), the experienced environmental changes are transmitted from mothers to their offspring, whose development is thereby altered. For example, mother daphniids reduce the size of their progeny in the presence of large-selective predators such as fish (Stibor, 1992), and do the opposite in the presence of small-size selective predators such as Chaoborus (Stibor and Lüning, 1994; Coors et al., 2004). OS may also vary in response to differing maternal food levels (Tessier and Consolatti, 1991; Glazier, 1992; Ebert, 1993; Enserink et al. 1993; Lampert, 1993; Guinnee, et al., 
Table 1. List of the different data sources used in the study and description of the experimental conditions using D. magna.

\begin{tabular}{|c|c|c|c|c|}
\hline$\overline{\text { Experiment (literature) }}$ & Clone & Density (mL.daphniid ${ }^{-1}$ ) & Food concentration (mg C.daphniid ${ }^{-1} . \mathrm{d}^{-1}$ ) & Scenarios \\
\hline a (Cleuvers, 1995) & 5 & $12.5-50$ & 0.1 & FT \\
\hline b (Popovic, 1996) & 5 & $12.5-50$ & 0.1 & FT \\
\hline c (Goser, 1997) & 5 & $1.25-80$ & $0.1 ; 1$ & FT \\
\hline d (Coors, 1999) & Tonne & $15-80$ & $0.05 ; 0.075 ; 0.1 ; 0.2$ & SS \\
\hline e (Agatz, unpublished) & 5 & 80 & 0.2 & SS \\
\hline
\end{tabular}

FT and SS stand for flow-through and semi-static scenarios, respectively.

2004, 2006) or to varying population densities (Gliwicz and Guisande, 1992; Goser and Ratte, 1994; Burns, 1995; Cleuvers et al. 1997; LaMontagne and McCauley, 2001).

In this study, we investigated the interactive effects of multiple maternal traits and environmental rearing conditions on the neonate body size in Daphnia magna. We then parameterized a multivariate model describing OS variation with these variables. Finally, we validated the resulting model using an independent dataset.

\section{Material and methods}

\section{Experimental dataset}

Data from previous experimental studies made at the Institute of Environmental Research, RWTH Aachen University (Cleuvers, 1995; Popovic, 1996; Goser, 1997; Coors, 1999; Agatz, unpublished; see Table 1) were used for parameterizing the model. Daphniids ( $\leq 24 \mathrm{~h}$ old) used in these tests originated from third broods of acclimated mothers. Mothers had been individually reared in cultures kept under constant temperature and light conditions $\left(20 \pm 1{ }^{\circ} \mathrm{C}\right.$, 16-h light: 8 -h dark photoperiod in a climatecontrolled chamber) for several generations (almost 30 years) in the laboratory. These cultures were fed three times a week with log-phase Desmodesmus subspicatus. The algae were harvested from batch cultures grown in the medium as described by Kuhl and Lorenzen (1964), centrifuged and resuspended in $80 \mathrm{~mL}$ Elendt M4 medium. Mothers belonged to two different clones (Table 1): clone 5 was reared under laboratory conditions, and clone Tonne originated from the field sampling near Aachen (Coors, 1999). Experiments a, b and c were conducted under flowthrough conditions at constant food levels and a flow rate of $12 \mathrm{~mL} \cdot \mathrm{min}^{-1}$ (according to Goser and Ratte, 1994). Experiments $\mathrm{d}$ and e were run under the semi-static scenario, where daphniids were fed daily and the culture medium changed every Monday, Wednesday and Friday.

\section{Measured variables}

We tested the following maternal traits as potential variables affecting the OS: the maternal body length and age, the brood size, the brood number and the amount of ingested carbon per mother. Food concentration and density-dependent effects on OS were tested as potential environmental variables influencing the OS.
Determining the maternal body length, age, brood number and brood size

Within each released brood, the maternal body length and age, as well the brood size and number were recorded, and the average OS per brood was calculated. The individual measurements of neonates and mothers' lengths were made within $12 \mathrm{~h}$ of the time of brood release. Body lengths were measured (from top of eye to base of posterior spine) using a software designed at the institute (Preuss, unpublished). The mother and all her released offspring were placed in a Petri dish using a pipette and the surplus of water was removed to prevent the movement of the animals. The Petri dish was scanned (Canoscan $8800 \mathrm{~F}$ ) at $\times 1200 \mathrm{dpi}$ resolution. Once the scan finished, the mother was transferred into the medium and the neonates were discarded.

\section{Calculating the ingested carbon per mother daphniid}

Because the experiments were run under different feeding scenarios (semi-static and flow-through), we used the ingested carbon for each individual mother as an explanatory variable for OS instead of the food concentration administered. To calculate the ingested carbon, we used a dynamic individual-based model for D. magna (Preuss et al., 2009), which was set to the environmental conditions of each experiment described in Table 1. A dynamic simulation is especially necessary for semi-static conditions, where food availability might be triggered by the ingestion rate of the daphniid and the volume of the beaker. The model dynamically simulates the ingestion of daphniids based on the equation of McMahon and Rigler (1963), including the maximum filtration rate, a half-saturation constant $\left(K_{\mathrm{s}}\right)$ and the incipient limiting level (ILL). This function describes the filtration rate of daphniids to be constant below the ILL at the maximum filtration rate and to decrease with increasing food concentration above the ILL (Lampert, 1987). This behaviour leads to a constant ingestion rate at higher food concentrations (Preuss et al., 2009). The maximum filtration rate for the daphniid is calculated as a function of body length. In this equation, feeding is expressed by means of daily ingested carbon per individual (IC, Equation (1)), which depends on the filtration rate in $\mathrm{mL} \cdot \mathrm{h}^{-1}(F)$ and on the food concentration $(C)$ in $\mathrm{mg} \mathrm{C.mL}{ }^{-1}$.

$$
I C=F \times C \times 24
$$


The filtration rate (Equation (2), Preuss et al., 2009) is calculated as a function of maternal body length $(L$, in $\mathrm{mm})$ and the concentration of algae $\left(c\right.$, in cells. $\left.\mathrm{mL}^{-1}\right)$.

$$
F=\frac{p \times L^{s} \times K_{\mathrm{s}}}{K_{\mathrm{s}}+c-\mathrm{ILL}}
$$

with $P=0.5 \mathrm{~mL} \cdot \mathrm{mm}^{\mathrm{s}} \cdot \mathrm{h}^{-1}$ : factor for filtration rate, $s=2.45$ : scaling factor for filtration rate, $K_{\mathrm{s}}=30644$ cells. $\mathrm{mL}^{-1}$ : half saturation constant, ILL $=8506$ cells. $\mathrm{mL}^{-1}$ : incipient limiting level.

\section{Data analysis}

Statistical analyses were made using SPSS software (IBM SPSS statistics version 20) and the graphs were plotted in Sigma Plot (Systat Software, Inc. Sigma Plot SPW 11.0). We examined the relationships between OS and all the measured variables mentioned in the previous sections.

\section{Variation of OS with the environmental variables: density-dependent effects}

Experiments a, b, c and d (Table 1) contained data from both control and density conditions, where the daphnids were reared in groups in the same beaker and the available volume per daphnid varied from 1.25 to $80 \mathrm{~mL}$ (Table 1). This dataset was used separately to build up the regression model relating OS variation to densitydependent effects. Determining this relationship was done stepwise: to discard food level effects, we considered data relative to only one food concentration $(0.1 \mathrm{mg}$ C.daphniid $\left.{ }^{-1} \cdot \mathrm{d}^{-1}\right)$. For each experiment, we used the data from 'control' daphniids to make linear regressions relating OS to maternal body size (relative to $0.1 \mathrm{mg}$ C.daphniid $\left.{ }^{-1} \cdot \mathrm{d}^{-1}\right)$. The obtained equations were used to derive the expected sizes of offspring released from mothers under density conditions. The average relative OS (measured/expected OS) was then plotted against the density values (expressed as available volume per daphnid).

\section{Variation of OS with the maternal traits}

In order to check for the existence of genetic (clonal) differences within our dataset, we ran an analysis of variance (ANOVA, $P<0.05$ ) where the homogeneity of variances (Levene's test) and the normality of the distribution (Shapiro-Wilk test) were verified.OS variation with the maternal body size was studied for the different food concentrations and regression analyses were made to assess the significance of the different relationships. Effects of brood size and brood number on OS were tested using simple linear regressions. Similarly to density-dependent effects, the relationship of OS to the ingested carbon was determined by deriving the mean expected OS from the regression equation relative to data for $0.2 \mathrm{mg} \mathrm{C}$ (see Fig. 1(a)). Then, the relative OS, which is a function of maternal body length, was plotted against the daily amount of ingested carbon.

\section{Multiple regression analysis}

Stepwise multiple linear regression analysis (MLR) was conducted in order to generate equations linking OS to effects of maternal traits and environmental factors (e.g., maternal body size, maternal age, brood size, ingested carbon and brood number). This approach accounts for the effect of each variable after controlling for the effects of other variables on OS. In these analyses, the independence of the errors, homoscedasticity and normality of errors were verified. The significance of maternal body size, age, brood size, brood number and the ingested carbon were tested. The accuracy of the obtained models was judged by the value of the coefficient of determination $\left(r^{2}\right)$ and the significance of each predictive variable ( $t$-test). Once the significant variables were identified, we tested the goodness of fit to the data by: (i) plotting the measured against the predicted data and determining the $r^{2}$, and (ii) determining the mean (Equation (3)) and the maximum deviation of the measured data to the model.

Deviation $(\%)=\frac{\text { Measured OS }- \text { Predicted OS }}{\text { Measured OS }} \times 100$

\section{Model validation}

The ability of the developed model to predict OS variation was tested against an independent dataset (Glazier, 1992) obtained from D. magna reproduction tests using two genetically distinct clones (L-F and P-S1). The culture medium (120 mL of ASTM hard water) as well as food source (Chlorella vulgaris) differed from the dataset used for developing the model. The experiments were carried out in $120 \mathrm{~mL}$ ASTM media with individual daphniids. Daphniids were daily fed $0.3 \mathrm{mg} \mathrm{C.L^{-1 }}$ $\left(0.036 \mathrm{mg}\right.$ C.daphniid $\left.{ }^{-1} . \mathrm{d}^{-1}\right)$ and $1.5 \mathrm{mg}$ C. $\mathrm{L}^{-1}$ $\left(0.18 \mathrm{mg}\right.$ C.daphniid $\left.{ }^{-1} \cdot \mathrm{d}^{-1}\right)$. The carbon content of C. vulgaris was transformed to its equivalent for D. subspicatus, assuming that D. subspicatus has an average carbon content of $1.95 \times 10^{-8} \mathrm{mg} \mathrm{C}$ cell ${ }^{-1}$ (SokullKluettgen, 1998; unpublished results from the Institute of Environmental Research, RWTH Aachen University). The data provided information on the egg dry mass. The neonate's dry weight (DW) was derived from egg dry mass using the relationships given in Glazier (1992: Table 3). Accordingly, newborns were $22.5 \%$ heavier than eggs for clone L-F and $47.05 \%$ for clone P-S1. Finally, a regression model (Kooijman, 2000) was fitted (Equation (4)) to convert the DW of neonates into body length $(\mathrm{mm})$ :

$$
\mathrm{DW}=11.89 \times \text { Length }^{3}
$$

\section{Results}

In our dataset, OS was not significantly influenced by clonal differences (ANOVA, $P>0.05$ ). 

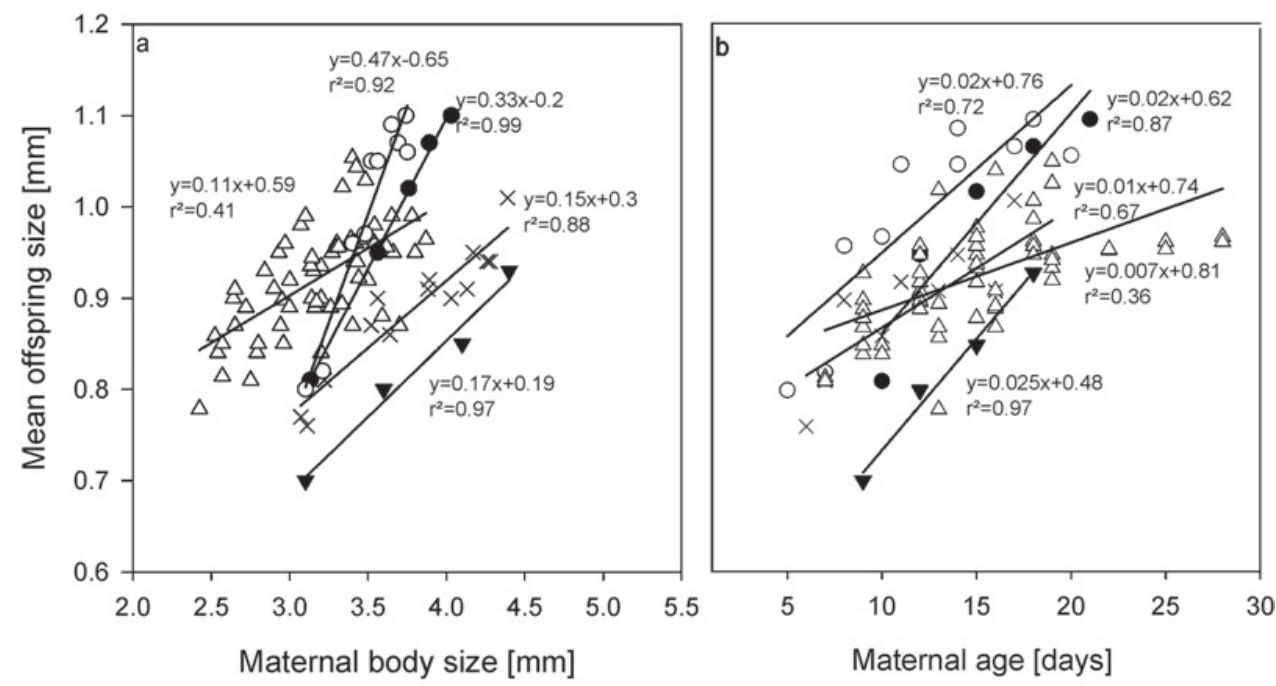

\begin{tabular}{|llllll|}
\hline$\circ$ & $0.05 \mathrm{mgC}$ & $\triangle$ & $0.1 \mathrm{mgC}$ & & $1 \mathrm{mgC}$ \\
$\bullet$ & $0.075 \mathrm{mgC}$ & $\times$ & $0.2 \mathrm{mgC}$ & & \\
\hline
\end{tabular}

Fig. 1. Dependence of the mean OS on the maternal body size (a) and age (b) in D. magna in relation to food level $(n=118)$.

\section{OS dependence on maternal traits}

\section{Effects of maternal body size and age}

OS showed significant positive linear relationships with maternal body size and age at all food concentrations (Fig. 1(a) and (b)). The slopes of the different regression equations linking OS to these two variables were in general steeper at low $(0.05$ and $0.075 \mathrm{mg} \mathrm{C})$ than higher food concentrations $(0.1-1 \mathrm{mg} \mathrm{C})$. Large females (body size $\geq 3.5 \mathrm{~mm}$ ) reared at high food concentrations produced smaller offspring than the low-fed ones of the same size (Fig 1(a)). However, for the smaller mothers (body size $\leq 3 \mathrm{~mm}$ ), OS had a non-linear response with food: it increased with decreasing food concentration from 1 to $0.1 \mathrm{mg} \mathrm{C}$ and then decreased when food decreased down to $0.05 \mathrm{mg}$ C. In comparison, females of the same age produced smaller offspring with increasing food concentration (Fig. 1(b)).

\section{Effects of the daily amount of ingested carbon}

Independently of the maternal body size, there was a significant negative relationship between OS and the ingested carbon $\left(r^{2}=0.52, n=118\right.$; Fig. 2$)$ : the largest neonates were born to mothers with the lowest ingested carbon and the smallest neonates to mothers with the highest ingested carbon.

\section{Effects of the brood size}

There were no data available on the brood size for daphnids reared at $1 \mathrm{mg} \mathrm{C}$. Based on the remaining dataset, OS tended to decrease with larger broods, but the relationship was not significant $\left(r^{2}=0.051, n=114\right.$; Fig. 3).

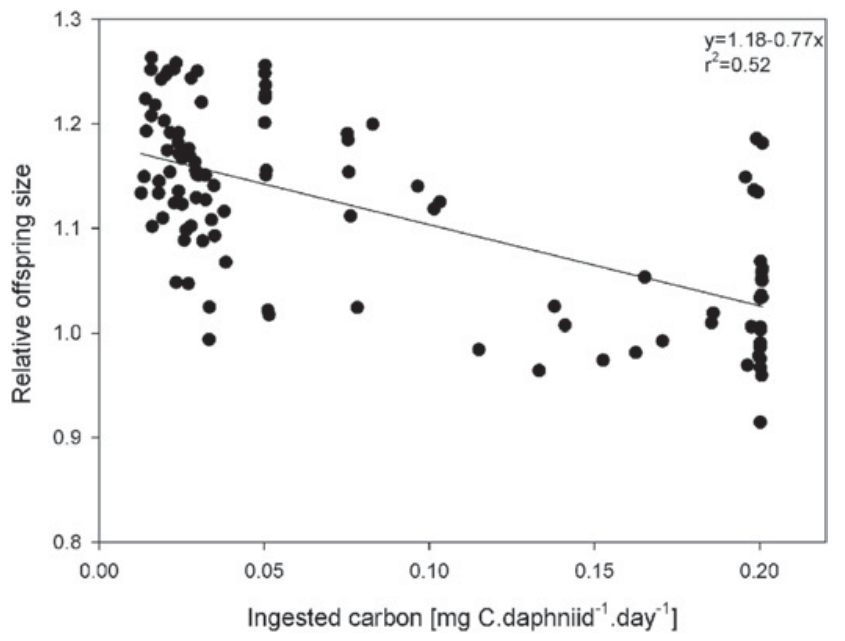

Fig. 2. Dependence of the relative OS on the daily amount of ingested carbon in D. magna. The relative OS is the ratio between measured values and model predictions based on the maternal body size.

\section{OS dependence on the maternal environment}

The variation in the relative OS (based on the regression of OS on maternal body length and the food concentration) with density followed a two-parameter exponential-decay function (Equation (5), Fig. 4).

$$
\begin{gathered}
\text { Relative offspring size }=1+a \times e^{-b \times \text { density }} ; \\
r^{2}=0.94 ; n=169
\end{gathered}
$$

with $a=0.1266 ; b=0.0659$; and density expressed in $\mathrm{mL}$ per daphniid.

OS varied significantly with density $(P<0.05)$. At minimal density values ranging between 80 and $50 \mathrm{~mL}$ per daphniid, there was no variation in the OS. 


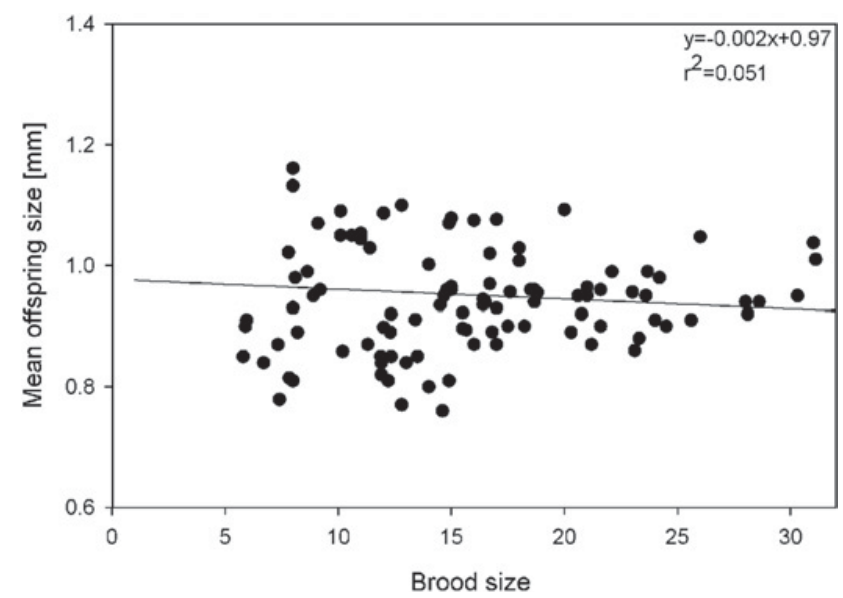

Fig. 3. Dependence of the mean OS on the brood size $(n=114)$ in D. magna (excluding the dataset for $1 \mathrm{mg} \mathrm{C}$ in reference c, Table 1).

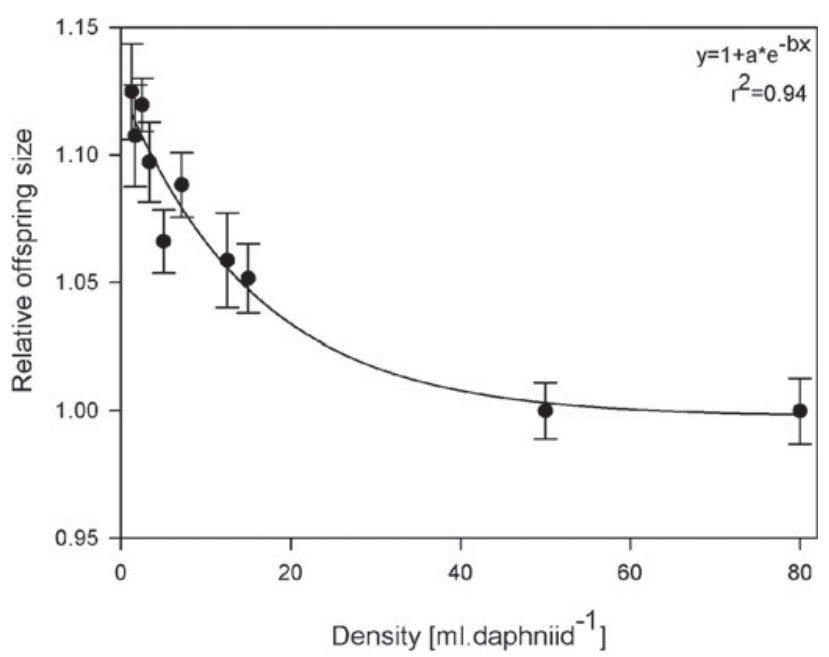

Fig. 4. Dependence of the relative OS on density in D. magna $(n=169)$. The relative $\mathrm{OS}$ is the ratio between measured values and model predictions based on maternal body size. Food effect was excluded by considering data deriving from only one food level $\left(0.1 \mathrm{mg}\right.$ C.daphniid $\left.{ }^{-1} \cdot \mathrm{d}^{-1}\right)$. Error bars indicate $95 \%$ confidence intervals.

However, the OS significantly increased with increasing densities. Density effects were most pronounced when the volume decreased below $20 \mathrm{~mL}$ per daphniid. Under these conditions, the predicted OS was significantly smaller than the observed OS.

\section{The multivariate model for OS}

The parameters obtained by stepwise MLR and their significance are summarized in Table 2. Effects of maternal body size, brood size and the ingested carbon were significant $(P<0.0001)$. Maternal age had a $P$ value of 0.039 , whereas brood number was not a significant vari- able $(P=0.185)$ and was removed from the model. OS showed a positive dependency on maternal body size and age, and negative dependencies on the ingested carbon and brood size. The model was able to describe $71 \%$ of the variability within the dataset.

Because maternal body size and age are strongly related variables, and age was the last variable introduced last in the MLR model, we ran another MLR excluding this variable. The results (Table 3 ) show that all variables included in the analysis contributed significantly to determining the OS. Moreover, by removing the maternal age, the collinearity between the explanatory variables was significantly reduced without affecting the model's goodness of fit $\left(r^{2}=0.7\right)$. Therefore, the final model describing OS variation with the maternal body size (ML), ingested carbon (IC) and brood size (BS) as explanatory variables is shown in Equation (6).

$$
\begin{gathered}
\mathrm{OS}=0.436+0.184 \times \mathrm{ML}-0.595 \times \mathrm{IC}-0.00567 \times \mathrm{BS} ; \\
r^{2}=0.7 ; n=114
\end{gathered}
$$

The model fitted well to the measured data (Fig. 5(a)) with a mean deviation of $4.01 \pm 3.2 \%$ and a maximum deviation of $16.92 \%$.

OS dependence on density was considered by multiplying the MLR by the non-linear density effect function. The model describing OS variation to density in addition to maternal body size, brood size and ingested carbon was obtained by multiplying the MLR equation (6) by the density-effect equation (5). The resulting equation is:

$$
\begin{aligned}
\mathrm{OS} & =[0.436+0.184 \times \mathrm{ML}-0.595 \times \mathrm{IC}-0.00567 \times \mathrm{BS}] \\
& \times\left(1+0.1266 \times e^{-0.0659 \times \text { density }}\right) ; \quad r^{2}=0.65 ; n=169
\end{aligned}
$$

The model described OS variation in a good agreement with the measured data $\left(r^{2}=0.65\right.$, Fig. $\left.5(\mathrm{~b})\right)$ with a mean and a maximum deviation of $4.07( \pm 3.16 \%)$ and $16.92 \%$, respectively.

\section{Model validation}

There were no validation data available for density dependence effects. Thus, the results in Figure 6 show the validation of the MLR model obtained under density-free conditions (Equation (6)), predicting the variation of OS with maternal body size, brood size and ingested carbon. The model appears to be largely validated because the measured OS varied with the predicted OS with a slope of 1.03 and an intercept of 0.007 , which should ideally equal 1 and 0 , respectively. The model was able to describe $36 \%$ of the variability in the data.

\section{Discussion}

In this work, we set up an empirical model describing the variation in the size of offspring born from mothers reared under different feeding and density conditions. The model was tested against an independent dataset using two 
Table 2. Multiple linear regressions of OS on maternal body size, maternal age, brood number, brood size and the ingested carbon. All dataset (except $1 \mathrm{mg} \mathrm{C}$ in experiment c) were used in this analysis.

\begin{tabular}{|c|c|c|c|c|c|}
\hline & Factors & Coefficient & Standard error & $t$-test & $P$ \\
\hline \multirow[t]{2}{*}{ Step one } & Constant & 0.630 & 0.031 & 20.560 & $<0.0001$ \\
\hline & Maternal body size & 0.090 & 0.008 & 10.776 & $<0.0001$ \\
\hline \multirow[t]{5}{*}{ Final step } & Constant & 0.495 & 0.045 & 11.052 & $<0.0001$ \\
\hline & Maternal body size & 0.156 & 0.018 & 8.599 & $<0.0001$ \\
\hline & Brood size & -0.005 & 0.001 & -6.758 & $<0.0001$ \\
\hline & Ingested carbon & -0.536 & 0.112 & -4.793 & $<0.0001$ \\
\hline & Age & 0.002 & 0.001 & 2.175 & 0.039 \\
\hline
\end{tabular}

Table 3. Multiple linear regressions of OS on maternal body size, brood size and the ingested carbon. All dataset (except $1 \mathrm{mg} \mathrm{C}$ in experiment c) were used in this analysis.

\begin{tabular}{|c|c|c|c|c|c|}
\hline & Factors & Coefficient & Standard error & $t$-test & $P$ \\
\hline \multirow[t]{2}{*}{ Step one } & Constant & 0.634 & 0.031 & 20.544 & $<0.0001$ \\
\hline & Maternal body size & 0.089 & 0.008 & 10.634 & $<0.0001$ \\
\hline \multirow[t]{4}{*}{ Final step } & Constant & 0.436 & 0.037 & 11.799 & $<0.0001$ \\
\hline & Maternal body size & 0.184 & 0.0134 & 13.722 & $<0.0001$ \\
\hline & Brood size & -0.00567 & 0.00079 & -7.185 & $<0.0001$ \\
\hline & Ingested carbon & -0.595 & 0.11 & -5.387 & $<0.0001$ \\
\hline
\end{tabular}
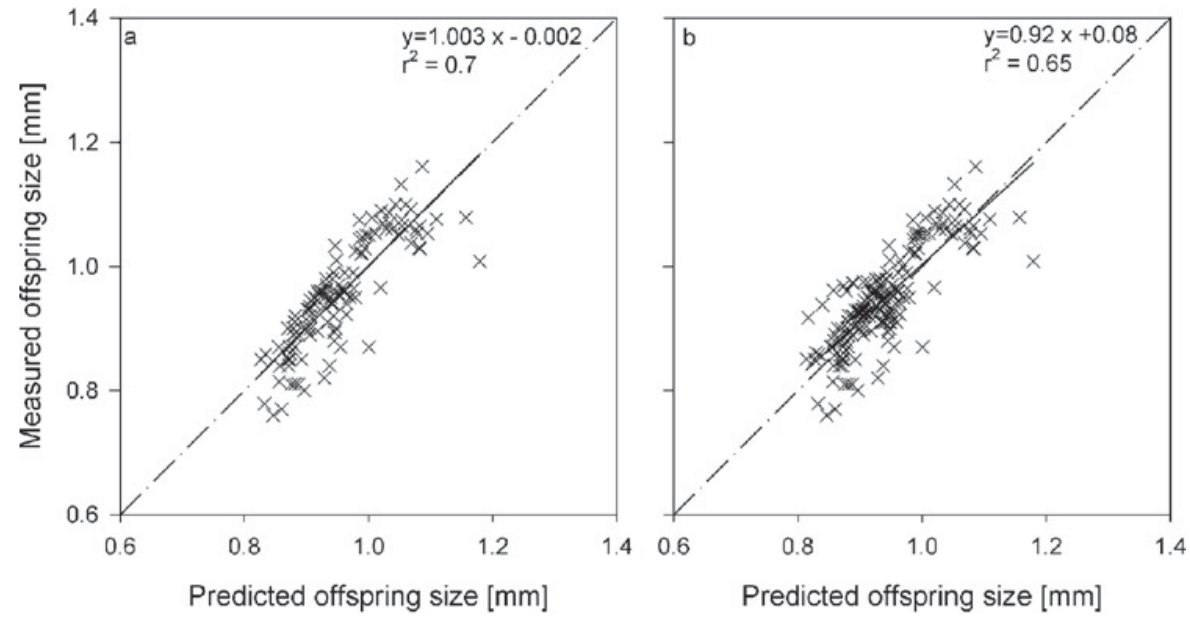

Fig. 5. Predicted-measured statistics for the OS (excluding the dataset for $1 \mathrm{mg} \mathrm{C}$ in reference c, Table 1). Predicted values were obtained by means of multiple linear regressions without $(\mathrm{a}, n=114)$ and with $(\mathrm{b}, n=169)$ density effects. Full lines show the regressions and dashed lines the optimal 1:1 prediction.

genetically different clones (which also differed from the clones used in the parameterization of the model), a different food source and culture medium, as well as different food levels. It is important to mention that all the allometric relationships of brood mass, brood size and egg mass were significantly different between the two clones (see Glazier, 1992 for details). Besides, if we used life-cycle data from flow-through and semi-static tests to develop the model, validation data were obtained at semi-static conditions. Despite these divergences, the multivariate model accurately described the variation in OS (Fig. 6).

The amount of ingested carbon was the most determining factor of OS variation, contrary to brood size which accounted for the least effects. Even though the two variables were correlated, maternal body size was a better predictor than maternal age. Additionally, the brood number did not significantly influence the OS and therefore, the exclusion of these two variables from the model did not affect its efficiency to describe the variation in OS. Our results support the findings of Ebert (1993), showing that age and brood number did not affect OS in two D. magna populations from different artificial ponds, but the food level, maternal body size and brood size strongly affected OS. However, opposite results were observed for D. galeata (Sakwinska, 2004) where the juvenile growth increment differed between young, intermediate and older mothers, leading to a dependence of OS on maternal age. The observed variability in the factors determining OS in Daphnia could be attributed to interspecies differences. 


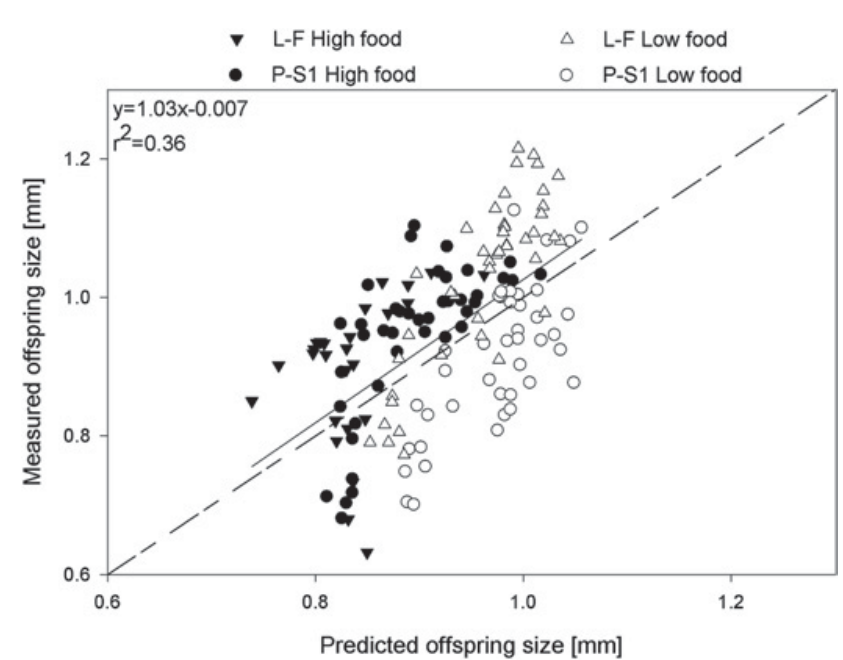

Fig. 6. Test of the model on independent data using two distinct clones: P-S1 and L-F, and two different food levels. Data $(n=83)$ originated from Glazier (1992). The full line indicates the model predictions and the dashed line the optimal 1:1 prediction.

\section{OS dependence on maternal body size}

Positive linear relationships between the size of the mother and that of her offspring were obtained under high, intermediate and low food levels. Other studies have shown similar relationships where the effort per offspring (in terms of size or mass of eggs or neonates) increased with maternal body size for different clones of D. magna, under high and low food conditions (Glazier, 1992; Ebert, 1993; McKee and Ebert, 1996). Positive effects of maternal body size on OS were observed in other Daphnia species such as D. galeata (Sakwinska, 2004) or D. hyalina (Burns, 1995) and in other organisms (Bernardo, 1996) as well.

\section{OS dependence on the ingested carbon}

Mothers clearly showed a dynamic shift in the way they provision their offspring when food decreases, changing from an emphasis on egg number in food-rich environments to egg size in food-poor environments. These results support the findings of Glazier's (1992) humped-shape model, predicting a positive relationship between OS and the ingested carbon at low food levels and a negative correlation at high food levels. The negative relationship observed in the present study is also found in other Daphnia species such as D. pulex (Taylor 1985), D. pulicaria and D. hyalina (Guisande and Gliwicz, 1992). By contrast, positive covariation between OS and food level was found in two different studies with $D$. pulex (Lynch, 1989; LaMontagne and McCauley, 2001). In these studies, either very low food concentrations were used (equivalent to 0.01-0.06 mg C per Daphnia per day, see LaMontagne and McCauley, 2001) or the daphnids were not daily fed as done in the present study or in Glazier (1992)'s study, which was used to validate our model.
These two experiments thus fit into the left-hand side of the humped-shape model.

\section{OS dependence on maternal body size and food level}

In large females (body size $\geq 3.5 \mathrm{~mm}$ ), OS showed a negative relationship with food concentration. In small females (body size $\leq 3 \mathrm{~mm}$ ), the increase in OS with decreasing food concentrations (down to $0.1 \mathrm{mg}$ C.daphniid $\left.{ }^{-1} . \mathrm{d}^{-1}\right)$ and its decrease at the lowest food concentrations (down to $0.05 \mathrm{mg} \mathrm{C}$.daphniid ${ }^{-1} . \mathrm{d}^{-1}$ ) are consistent with the humped-shaped model of Glazier (1992, Fig. 7), where both positive and negative relationships between OS and food demand are hypothesized. At very low food levels, egg mass becomes smaller in Daphnia because very small females are structurally and energetically incapable of producing large eggs (Glazier, 1992). This hypothesis may also explain the steeper slopes of the regressions relating OS to maternal body length at the lowest food levels: under these conditions, small females were constrained to produce small offspring because of energy constraints and the spatial limitations in the brood pouch. However, large females may have had enough energy reserves to show the adaptive response of producing relatively large offspring, as was observed in other Daphnia studies (Glazier, 1992; Ebert, 1993; Lampert, 1993; Boersma, 1995, 1997). At higher food levels, the slope was less steep because small females were not as energy limited, and large females were favoured to produce many small offspring. In this way, small daphniids continued to produce smaller offspring than the larger ones because of spatial limitations of the brood pouch. However, the difference was not as great because energy limitation is less important, resulting in the observed shallower slopes. The results obtained in this study support optimal offspring investment theory, which predicts that larger offspring should be produced under low compared with high food conditions (Goulden et al., 1987), as long as the mothers are not too small (thus preventing them from making larger offspring, as predicted by Glazier's (1992) OS response model).

Our analysis of the OS with both maternal body length and food concentration showed that, even at a laboratory scale where it is purposely attempted to reduce experimental variability, mother daphniids have different reproductive strategies according to their interactions with the environment, which had important repercussions on OS.

\section{OS dependence on brood size}

OS was larger in small broods compared with large ones. Most studies observed the same pattern in D. magna (Ebert, 1993; Boersma, 1997) and other cladoceran species (Taylor, 1985; Gliwicz and Guisande, 1992). However, brood size accounted for only a small proportion of variability compared with other factors; i.e., the ingested carbon and the maternal body size. The importance of this variable in determining OS might 
be indirectly related to its interaction with other variables, such as the food level (Ebert, 1993).

\section{OS dependence on density}

It is well known that Daphnia changes its reproductive strategy under varying density conditions. At high densities, daphniids grow more slowly and produce fewer offspring (Guisande, 1993; Goser and Ratte, 1994). Density effects were also shown to propagate to the $F_{1}$ generation whereby daphniids living singly but descending from ancestors living in groups produced significantly larger offspring $\left(\mathrm{F}_{2}\right)$ than daphniids descending from singly living ancestors (Cleuvers et al., 1997). Our results show that the daphniids responded to increasing density conditions by an increase in OS at the expense of brood size which decreased. This was observed by disentangling the food level effects. Our results are similar to those obtained by other authors (Cox et al., 1992; Naylor et al., 1992) who observed that at low densities, D. magna produces more and smaller neonates than at higher stocking densities where there were fewer and larger neonates produced. Cleuvers et al. (1997) explained that the daphniids shift their reproductive strategy from producing a higher quantity to a higher quality of neonates (heavier offspring) when the available culture volume is minimal. The increase in OS with increasing density conditions was also observed for other daphniid species such as D. pulex (Ban et al., 2009), other Daphniidae species such as Simocephalus vetulus (Perrin, 1989), as well as other aquatic invertebrates such as copepods (Cooney and Gehrs, 1980). However, contrasting results were recorded for other Daphnia species: Burns (1995) showed that for D. hyalina and $D$. galeata, mothers kept in density conditions $(\geq 150$ individuals per litre) produced smaller offspring and smaller broods.

\section{Adaptive value}

In addition to the strong relationships between OS and maternal life-history traits, mothers were able to change their reproductive strategy in accordance to changes in the environmental conditions, i.e., available food and density, and this shift was manifested by a change in OS. The observed patterns of OS variation with maternal traits and environmental factors suggest an adaptive shift from quantity to quality of offspring as food availability per individual decreases. During spring and early summer, Daphnia populations grow rapidly (Hülsmann, 2003; Wagner et al., 2004), resulting in a depletion of available resources. At the end of the spring algal bloom, newborn daphniids have few available resources, exerting high physiological stress on individuals, which results in an elevated non-consumptive mortality (Hülsmann, 2003). These processes lead to a declining population size of Daphnia, which in some cases, particularly in eutrophic waters, can directly proceed to the initiation of a midsummer decline of daphniids (Hülsmann and Weiler,
2000; Hülsmann, 2003). In this context, producing fewer larger (fitter) offspring at low food levels and high population densities, but many small offspring at high food levels and low population densities may be adaptive responses for increasing population survival and growth, respectively.

\section{Conclusion}

Our study shows that multiple maternal and environmental variables significantly affect OS in D. magna. As a result, future models addressing ecological or biological questions regarding Daphnia populations should include the natural variability of OS in relation to relevant maternal and environmental variables. This would ensure a more realistic prediction of individual behavior, thereby leading to a more accurate characterization of Daphnia population dynamics under natural or stressed conditions.

Acknowledgements. This work has been financially supported by the European Union under the 7th Framework Programme (Project acronym CREAM, contract number PITN-2009238148). The authors thank Dr. Udo Hommen and the anonymous reviewers for their valuable comments on previous versions of the manuscript.

\section{References}

Ban S., Hideaki T., Tsukasa M. and Nishimura K., 2009. Effects of physical interference on life history shifts in Daphnia pulex. J. Exp. Biol., 212, 3174-3183.

Barata C. and Baird D.J., 1998. Phenotypic plasticity and constancy of life-history traits in laboratory clones of Daphnia magna Straus: effects of neonatal length. Funct. Ecol., 12, 442-452.

Bernardo J., 1996. The particular maternal effect of propagule size, especially egg size: patterns, models, quality of evidence and interpretations. Amer. Zool., 36, 216-236.

Boersma M., 1995. The allocation of resources to reproduction in Daphnia galeata: against the odds? Ecology, 76, 1251-1261.

Boersma M., 1997. Offspring size and parental fitness in Daphnia magna. Evol. Ecol., 11, 439-450.

Burns C.W., 1995. Effects of crowding and different food levels on growth and reproductive investment of Daphnia. Oecologia, 101, 234-244.

Cleuvers M., 1995. Die Auswirkungen der negativen Interferenz auf die $\mathrm{F}_{1}$-Generation von Daphnia magna STRAUS. $\mathrm{PhD}$ Thesis, Rheinisch-Westfäalischen Technischen Hochschule Aachen, Germany.

Cleuvers M., Goser B. and Ratte H.T., 1997. Life-strategy shift by intraspecific interaction in Daphnia magna: change in reproduction from quantity to quality. Oecologia, 110, 337-345.

Cooney J.D. and Gehrs C.V.V., 1980. The relationship between egg size and naupliar size in the calanoid copepod Diaptomus clavipes Schacht. Limnol. Oceanogr., 25, 549-552. 
Coors A., 1999. Lebensänderung bei Daphnia magna als Reaktion auf verschiedene Umweltfaktoren unter dem Einfluss eines Dispergiermittels. PhD thesis, RheinischWestfäalischen Technischen Hochschule Aachen Germany.

Coors A., Hammers-Wirtz M. and Ratte H.T., 2004. Adaptation to environmental stress in Daphnia magna simultaneously exposed to a xenobiotic. Chemosphere, 56, 395-404.

Cox E.J., Naylor C., Bradley M.C. and Calow P., 1992. Effect of differing maternal ration on adult fecundity and offspring size in laboratory cultures of Daphnia magna Straus for ecotoxicological testing. Aquat. Toxicol., 24, 63-74.

Dudycha J.L. and Lynch M., 2005. Conserved ontogeny and allometric scaling of resource acquisition and allocation in the Daphniidae. Evolution, 59, 565-576.

Ebert D., 1993. The trade-off between offspring size and number in Daphnia magna: the influence of genetic, environmental and maternal effects. Arch. Hydrobiol., 90, 453-473.

Enserink L., de la Haye M. and Maas H., 1993. Reproductive strategy of Daphnia magna: implications for chronic toxicity tests. Aquat. Toxicol., 25, 111-123.

Gergs A. and Ratte H.T., 2009. Predicting functional response and size selectivity of juvenile Notonecta maculata foraging on Daphnia magna. Ecol. Model., 23, 3331-3341.

Glazier D.S., 1992. Effects of food, genotype and maternal size on offspring investment in Daphnia magna. Ecology, 73, 910-926.

Gliwicz Z.M. and Guisande C., 1992. Family planning in Daphnia: resistance to starvation in offspring born to mothers grown at different food levels. Oecologia, 91, 463-467.

Gergs A., Zenker A., Grimm V. and Preuss T.G., 2013. Chemical and natural stressors combined: from cryptic effects to population extinction. Scientific Reports (Nature Publishing Group), 3, 2036, DOI: 10.1038/srep02036.

Goser B., 1997. Dichteabhängige Änderungen der Entwicklung und Reproduktion bei Cladoceran. $\mathrm{PhD}$ thesis. Westfäalischen Technischen Hochschule Aachen Germany.

Goser B. and Ratte H.T., 1994. Experimental evidence of negative interference in Daphnia magna. Oecologia, 98, 354-361.

Goulden C.E., Henry L.L. and Berrigan D., 1987. Egg size, postembryonic yolk and survival ability. Oecol. (Berl.), 72, $28-37$.

Guinnee M.A., West S.A. and Little T.J., 2004. Testing small clutch size models with Daphnia. Amer. Nat., 163, 880-887.

Guinnee M.A., Gardner A., Howard A.E., West S.A. and Little T.J., 2006. The causes and consequences of variation in offspring size: a case study using Daphnia. J. Evol. Biol., 20, $577-587$.

Guisande C., 1993. Reproductive strategy as population density varies in Daphnia magna (Cladocera). Freshwat. Biol., 29, 463-467.

Guisande C. and Gliwicz Z.M., 1992. Egg size and clutch size in two Daphnia species grown at different food levels. J. Plankton Res., 14, 997-1007.

Hammers-Wirtz M. and Ratte H.T., 2000. Offspring fitness in Daphnia: Is the Daphnia reproduction test appropriate for extrapolating effects on the population level? Environ. Toxicol. Chem., 19, 1856-1866.

Hülsmann S., 2003. Recruitment patterns of Daphnia: a key for understanding midsummer declines? Hydrobiologia, 491, 35-46.
Hülsmann S. and Weiler W., 2000. Adult, not juvenile mortality as a major reason for the midsummer decline of a Daphnia population. J. Plankton Res., 22, 151-168.

Kooijman S.A.L.M., 2000. Dynamic Energy and Mass Budgets in Biological Systems. Cambridge University Press, Great Britain.

Kuhl A. and Lorenzen H., 1964. Handling and culturing of Chlorella. In: Prescot D.H. (ed.), Methods in Cell Physiology. Academic, New York, NY, USA.

LaMontagne J.M. and McCauley E., 2001. Maternal effects in Daphnia: what mothers are telling their offspring and do they listen? Ecol. Lett., 4, 64-71.

Lampert W., 1987. Feeding and nutrition in Daphnia. In: De Marchi (ed.), Daphnia. Memorie dell'istituto Italiano Di Idrobiologia. Verbania Pallanza, Italy, pp. 461-482.

Lampert W., 1993. Phenotypic plasticity of the size at first reproduction in Daphnia: the importance of maternal size. Ecology, 74, 1455-1466.

Lynch M., 1989. The life history consequences of resource depression in Daphnia pulex. Ecology, 70, 246-247.

Mckee D. and Ebert D., 1996. The interactive effects of temperature, food level and maternal phenotype on offspring size in Daphnia magna. Oecologia, 107, 189-196.

McMahon J.W. and Rigler F.H., 1963. Mechanisms regulating feeding rate of Daphnia magna Straus. Can. J. Zool., 41, 321-327.

Mousseau T.A. and Fox C.W., 1998. The adaptive significance of maternal effects. Trends Ecol. Evol., 13, 403-407.

Naylor C., Cox E.J., Bradley M.C. and Calow P., 1992. Effect of differing maternal food ration on susceptibility of Daphnia magna Straus neonates to toxic substances. Aquat. Toxicol., 24, 75-82.

Perrin N., 1989. Population density and OS in the cladoceran Simocephalus vetulus (Müller). Funct. Ecol., 3, 29-36.

Popovic P., 1996. Ist die negative Interferenz ein allgemeines Phänomen bei Cladoceren? $\mathrm{PhD}$ thesis. Westfäalischen Technischen Hochschule Aachen, Germany.

Preuss T.G., Hammers-Wirtz M., Hommen U., Rubach M.N. and Ratte H.T., 2009. Development and validation of an individual based Daphnia magna population model: the influence of crowding on population dynamics. Ecol. Model., 220, 310-329.

Preuss T.G., Hammers-Wirtz M, Ratte HT, 2010. The potential of individual based population models to extrapolate effects measured at standardized test conditions to relevant environmental conditions-an example for 3,4-dichloroaniline on Daphnia magna. J. Environ. Monit., 12(11), 2070-2079.

Rinke K., 2006. Species-oriented model approaches to Daphnia spp.: linking the individual level to the population level. PhD thesis. Technische Universität Dresden, Germany.

Sakwinska O., 2004. Persistent maternal identity effects on life history traits in Daphnia. Oecologia, 138, 379-386.

Stibor H., 1992. Predator induced life-history shifts in a freshwater Cladoceran. Oecologia, 92(2), 162-165.

Stibor H. and Lüning H.T., 1994. Predator-induced phenotypic variation in the pattern of growth and reproduction in Daphnia hyalina (Crustacea: Cladocera). Funct. Ecol., 8, 97-101. 
Taylor B.E., 1985. Effects of food limitation on growth and reproduction of Daphnia. Arch. Hydrobiol., 21, 285-296.

Tessier A.J. and Consolatti N.L., 1991. Resource quantity and offspring quality in Daphnia. Ecology, 72, 468-478.

Vanoverbeke J., 2008. Modelling individual and population dynamics in a consumer resource system: Behavior under food limitation and crowding and the effect on population cycling in Daphnia. Ecol. Model., 216, 385-401.

Wagner A., Hülsmann S., Dörner H., Janssen M., Kahl U., Mehner T. and Benndorf J., 2004. Initiation of the midsummer decline of Daphnia as related to predation, non-consumptive mortality and recruitment: a balance. Arch. Hydrobiol., 160, 1-23. 